\title{
RESEARCH
}

\section{Clarity and diagnostic quality of digitized conventional intraoral radiographs}

\author{
$\mathrm{R} \mathrm{Goga}^{*, 1}$, NP Chandler ${ }^{1}$ and RM Love ${ }^{2}$ \\ ${ }^{1}$ Department of Oral Rehabilitation, School of Dentistry, University of Otago, Dunedin, New Zealand; ${ }^{2}$ Department of Oral Diagnostic \\ and Surgical Sciences, School of Dentistry, University of Otago, Dunedin, New Zealand
}

\begin{abstract}
Objectives: To compare digital images of conventional radiographs with the original radiographs for perceived clarity of endodontic files, periapical lesions and carious lesions, and to establish the diagnostic value of the digital images.

Methods: Four groups of ten radiographs were used: standardized bitewings demonstrating carious lesions, periapical radiographs of apical lesions, periapical radiographs showing endodontic files of various sizes at working length, and standardized periapical radiographs with size 08 files at working length. Radiographs were photographed using an Olympus C 2500-L digital camera and a Nikon D1X digital camera and were scanned using a Nikon Supercoolscan 4000 ED film/slide scanner. The digital images were then transferred to a Toshiba Satellite 2210 laptop. Three general dental practitioners compared each conventional radiograph with the three matching digital images. Images were ranked for clarity and were assessed for diagnostic quality. Data were analysed using General Estimating Equations.

Results: The clarity and diagnostic quality of the conventional radiographs were superior to the digital images produced by the three techniques $(P<0.001)$. No significant difference was found between the Nikon D1X and Camedia 2500-L cameras for clarity or diagnostic quality. The scanner was equivalent to the Camedia 2500-L camera for diagnostic quality, but was otherwise inferior to both cameras.

Conclusions: Digitizing conventional radiographs using current high-grade digital cameras or scanners does not produce images of diagnostic quality. Improved resolution of viewing monitors is necessary to fully harness the potential of digital technology.
\end{abstract}

Dentomaxillofacial Radiology (2004) 33, 103-107. doi: 10.1259/dmfr/13010370

Keywords: radiography, dental, digital; photography

\section{Introduction}

Digital radiography is becoming popular and since the early 1990s a number of studies have compared conventional periapical radiographs with digitized radiographs. Sanderink et $\mathrm{al}^{1}$ concluded that direct digital radiography (DDR) using the RadioVisioGraphy (RVG) system (Trophy, Paris, France) was comparable with conventional radiography for determining root canal length using ISO size 15 files but inferior to conventional images when size 10 files were used. However, Garcia et $\mathrm{al}^{2}$ found that there were no statistically significant differences between DDR using the Sens-A-Ray system (AMPAC Dental, Rockdale, Australia)

*Correspondence to: Radu Goga, Department of Oral Rehabilitation, School of Dentistry, University of Otago, PO Box 647, Dunedin, New Zealand;

E-mail: gogaradu@hotmail.com

Received 1 June 2003; revised 23 February 2004; accepted 23 February 2004 and conventional radiography when estimating endodontic working length. This is also applicable to the Digora (Soredex Orion Corporation, Helsinki, Finland) photostimulable storage phosphor luminescence imaging system. ${ }^{3}$ Recently, DDR was found to be comparable with conventional E-speed films in estimating lengths of canals with various curvatures when using built-in measuring software, although the image quality could be further optimized. ${ }^{4}$ The clarity of a digital image, however, is also an important consideration for clinical practice, and even with a radiopaque contrast medium, Digora digital image modes were inferior to conventional radiographs for assessing root canal anatomy. ${ }^{5}$ Similarly, when looking at the perceived clarity of an endodontic file tip and of periapical lesions, Digora images were significantly inferior to conventional films. ${ }^{6}$ Scanned digital images of conventional radiographs were 
also inferior to the conventional radiographs when looking at size 06 K-files. ${ }^{7}$

While digitization of conventional radiographs and their comparison with the digitized images has been undertaken and published, a review of the literature showed no such studies using digital cameras as the means of digitization. ${ }^{8,9}$ Nevertheless, digital cameras have been used to digitize conventional radiographs, using the same image sensor technology as DDR - the charge-coupled device (CCD) - to acquire the image. ${ }^{10-12}$ The advantages of digital camera technology producing images of diagnostic quality would be several. First, it would make use of a clinical tool that is increasingly in use around the world. Second, digital photographs are suited for immediate data transmission, for instance via e-mail to a specialist colleague or dental laboratory. They can also be instantly integrated into dental practice software and stored. Third, the digital camera image could be diagnostically enhanced in a similar fashion to digital radiographs, using existing camera software. Fourth, a dental practice that is becoming totally computerized could use a digital camera as a means of transferring patient records (conventional radiographs) onto the practice database. Finally, use of digital photographs greatly enhances patient education and acceptance of treatment.

The aim of this study was to compare the quality of digital scans and photographs of conventional radiographs with the original radiographs for the perceived clarity of endodontic files, periapical and carious lesions, and to establish the diagnostic value of the digital images. The quality of digital photographs taken by a low-end digital camera was also compared with those from a professional digital camera and a top quality slide/film scanner.

\section{Materials and methods}

Forty radiographs were divided into four groups of ten. Ten radiographs were bitewings taken using a Combex DX-907 X-ray machine (Belmont, NJ) operating at $10 \mathrm{~mA}$ and $70 \mathrm{kVp}$. These showed carious lesions and were standardized by using a Kwik-Bite film holder (Hawe Neos Dental, Bioggio, Switzerland). Radiographs were developed using an All Pro 2000M automatic developer (All-Pro Imaging, Hicksville, USA). Ten radiographs were images of periapical lesions, from a collection employed in student teaching exercises. Another ten radiographs were periapical films showing root canal files of various sizes at or close to endodontic working length. The final group of radiographs consisted of films with ISO size 08 files at or close to working length. These were taken as part of treatment using a Snapex paralleling device/film holder (Dentsply Rinn, IL) and a Source I DX 066 X-ray machine (Belmont, NJ) at $10 \mathrm{~mA}$ and $70 \mathrm{kVp}$. Developing was done with a Peri Pro 111 automatic developer (All-Pro Imaging, Hicksville, USA). The original radiographs were intentionally selected to provide the evaluators with images of satisfactory quality for the area of interest. Informed consent was obtained where necessary prior to the use of the images in the study.
The 40 radiographs were placed in slide holders on a light viewing box (Magni Viewer III, Proyrex, Tokyo, Japan) and photographed using an Olympus Camedia 2500-L digital camera (Olympus Optical Co. Ltd., Tokyo, Japan) and a Nikon D1X digital camera (Nikon Corporation, Tokyo, Japan). A 43-55 mm step-up ring and Olympic IS/L B-Macro lens was used with the Olympus camera, and a $60 \mathrm{~mm}$ Macro Nikkor lens with the Nikon camera. The Olympus camera has a CCD of 2.5 million pixels with a recording image of $1712 \times 1368$ pixels, and the Nikon camera features a CCD of 5.47 million pixels with a recording image of $3008 \times 1960$ pixels. The pixel values for both cameras are true values, that is, without interpolation.

The Nikon camera images were taken at the same camera-radiograph distance using a specially designed jig in use at the Medical Photography Unit. The Olympus images were taken with the camera flush with the surface of the slide holders; the Olympus camera was incompatible with the jig and the Nikon could not be used so close-up. Identical illumination was used throughout with the radiographs placed at the same location on the light box. The digital camera images were saved as JPEG files.

The mounted radiographs were also scanned with a Nikon Supercoolscan 4000 ED film/slide scanner (Nikon Corporation, Tokyo, Japan). This has a 23 million pixel CCD and a recording image of $3529 \times 5379$ pixels. Scanner images were saved as TIFF files.

Olympus digital camera images each had approximately $300 \mathrm{kB}$ of data, Nikon digital camera images had around $1 \mathrm{MB}$ of data and Nikon digital scanner images had about $10 \mathrm{MB}$ of data.

Digital images were loaded directly onto a Toshiba Satellite 2210 laptop (Toshiba Corporation, Tokyo, Japan) or transferred via a CD. The images were opened using Olympus Camedia Master software and, for the digital cameras, converted to 256 grey scale. For each conventional radiograph, the three corresponding digital images were then loaded on one slide using the Microsoft PowerPoint 1997 slide presentation program (Microsoft Corporation, Redmond, WA). Each slide used the full screen with a black background and had three equally-sized digital images.

Three experienced general dental practitioners were asked to compare each of the conventional radiographs with the three matching digital images and to rank the images in decreasing order of clarity (for their respective purpose). Also, each image (conventional or digital) was judged as to whether it was of adequate diagnostic quality for the purpose it was taken for. The evaluators were given only a brief period to become accustomed to the digital images prior to evaluating them.

The 40 conventional radiographs were examined on the light viewing box and the 120 digital images were viewed directly on the laptop monitor, with each digital image approximately nine times larger than the original radiograph. Image modification such as changing of contrast was not allowed. Image sequencing was the same for all evaluators. Two of the dentists used correcting lenses for their eyesight, while magnification from personal 
magnifying loupes, ranging from $2.5 \times$ to $3.5 \times$, was freely employed by all evaluators (although not for all images). Lux-meter readings of the light-box, performed throughout the photographic and evaluating process to ensure consistent output, were between 5200 lux and 5400 lux.

After 3 weeks, 16 randomly chosen images (four from each group) were assessed again under the same conditions to investigate intraexaminer reliability.

Statistical analysis was done using General Estimating Equations, with the level of significance being set at $P=0.05$. Kappa tests were used to determine intraexaminer and interexaminer agreement. Kappa values of 0.81 to 1.00 signify very good agreement, 0.61 to 0.80 good agreement, and 0.41 to 0.60 moderate agreement.

\section{Results}

Table 1 shows the initial readings for the 40 images and Table 2 shows the readings for the follow-up images.

The clarity and diagnostic quality of the conventional radiographs was superior to the digital images produced by the three techniques $(P<0.001)$. There was no statistically significant difference between the Nikon and Olympus camera images for clarity or diagnostic quality. The scanner images were equivalent to the Olympus camera images $(P=0.48)$ for diagnostic quality but otherwise inferior to both camera images in all other respects.

There was close to $100 \%$ intraexaminer and interexaminer agreement that the conventional radiographs

Table 1 Comparison of conventional radiographs with digital images

\begin{tabular}{|c|c|c|c|c|c|}
\hline Type of image & Rank 1 & Rank 2 & Rank 3 & Rank 4 & Diagnostic quality (max. 30) \\
\hline \multicolumn{6}{|c|}{ Ranking of bitewings (120 total rankings, 30 per type of image) } \\
\hline $\mathrm{R}$ & 30 & 0 & 0 & 0 & 29 \\
\hline S & 0 & 14 & 5 & 11 & 13 \\
\hline $\mathrm{O}$ & 1 & 15 & 9 & 8 & 11 \\
\hline $\mathrm{N}$ & 1 & 14 & 7 & 8 & 12 \\
\hline \multicolumn{6}{|c|}{ Ranking of periapical lesions (120 total rankings, 30 per type of image) } \\
\hline $\mathrm{R}$ & 30 & 0 & 0 & 0 & 30 \\
\hline $\mathrm{S}$ & 0 & 12 & 3 & 15 & 16 \\
\hline $\mathrm{O}$ & 3 & 15 & 8 & 4 & 17 \\
\hline $\mathrm{N}$ & 0 & 15 & 13 & 2 & 18 \\
\hline \multicolumn{6}{|c|}{ Ranking of various file sizes at working length ( 120 total rankings, 30 per type of image) } \\
\hline $\mathrm{R}$ & 30 & 0 & 0 & 0 & 27 \\
\hline $\mathrm{S}$ & 2 & 12 & 8 & 8 & 7 \\
\hline $\mathrm{O}$ & 3 & 16 & 7 & 4 & 11 \\
\hline $\mathrm{N}$ & 3 & 18 & 7 & 2 & 18 \\
\hline \multicolumn{6}{|c|}{ Ranking of ISO size 08 files at working length ( 120 total rankings, 30 per type of image) } \\
\hline $\mathrm{R}$ & 30 & 0 & 0 & 0 & 21 \\
\hline S & 0 & 7 & 10 & 13 & 10 \\
\hline $\mathrm{O}$ & 1 & 18 & 6 & 5 & 11 \\
\hline $\mathrm{N}$ & 2 & 21 & 6 & 1 & 12 \\
\hline
\end{tabular}

$\mathrm{R}$, original radiograph; $\mathrm{S}$, Nikon scanner image; O, Olympus camera image; N, Nikon camera image

Table 2 Follow-up comparison after 3 weeks

\begin{tabular}{|c|c|c|c|c|c|}
\hline Type of image & Rank 1 & Rank 2 & Rank 3 & Rank 4 & Diagnostic quality (max. 12) \\
\hline \multicolumn{6}{|c|}{ Ranking of bitewings ( 48 total rankings, 12 per type of image) } \\
\hline $\mathrm{R}$ & 12 & 0 & 0 & 0 & 12 \\
\hline S & 0 & 4 & 5 & 3 & 8 \\
\hline $\mathrm{O}$ & 0 & 8 & 4 & 0 & 8 \\
\hline $\mathrm{N}$ & 0 & 3 & 1 & 8 & 3 \\
\hline \multicolumn{6}{|c|}{ Ranking of periapical lesions (48 total rankings, 12 per type of image) } \\
\hline $\mathrm{R}$ & 12 & 0 & 0 & 0 & 12 \\
\hline $\mathrm{S}$ & 1 & 6 & 2 & 3 & 9 \\
\hline $\mathrm{O}$ & 0 & 8 & 3 & 1 & 9 \\
\hline $\mathrm{N}$ & 0 & 5 & 4 & 3 & 9 \\
\hline \multicolumn{6}{|c|}{ Ranking of various file sizes at working length ( 48 total rankings, 12 per type of image) } \\
\hline $\mathrm{R}$ & 12 & 0 & 0 & 0 & 12 \\
\hline $\mathrm{S}$ & 1 & 4 & 3 & 4 & 4 \\
\hline $\mathrm{O}$ & 3 & 7 & 1 & 1 & 8 \\
\hline $\mathrm{N}$ & 2 & 6 & 2 & 2 & 7 \\
\hline \multicolumn{6}{|c|}{ Ranking of ISO size 08 files at working length ( 48 total rankings, 12 per type of image) } \\
\hline $\mathrm{R}$ & 12 & 0 & 0 & 0 & 11 \\
\hline S & 1 & 4 & 2 & 5 & 4 \\
\hline $\mathrm{O}$ & 2 & 4 & 3 & 3 & 6 \\
\hline $\mathrm{N}$ & 3 & 6 & 1 & 2 & 5 \\
\hline
\end{tabular}

$\mathrm{R}$, original radiograph; $\mathrm{S}$, Nikon scanner image; O, Olympus camera image; $\mathrm{N}$, Nikon camera image 
were superior to all the digital images; this very high agreement precluded obtaining a Kappa value. There was also very high intraexaminer agreement that the conventional radiographs were of diagnostic quality, precluding obtaining a Kappa value. The interexaminer agreement that the conventional radiographs were of diagnostic quality was $87 \%$, with a Kappa value of 0.48 .

Out of 120 assessments, a digital imaging modality ranked equal to the radiograph only 10 times. The radiograph was considered to be diagnostic 103 times and the Nikon camera image, Olympus camera image and Nikon scanner image 60, 50 and 45 times, respectively. There were no incidences where a digital image was considered diagnostic if the radiograph was not considered to be so.

There was no firm intraexaminer agreement (Kappa values from -0.09 to 0.08 ) or interexaminer agreement (Kappa values from -0.03 to 0.07 ) on whether one digital imaging system produced better images than the others. Likewise, there was no firm intraexaminer agreement (Kappa values from -0.09 to 0.08 ) or interexaminer agreement (Kappa values from -0.13 to 0.15 ) on whether the digital images were of diagnostic quality.

\section{Discussion}

Digital technology promises and delivers much to both dentistry and medicine. For many applications, including the dental operating microscope, the image quality of digital photographs is comparable with that of conventionally taken photographs. ${ }^{13,14}$ Consumer digital cameras are now specified as having 3-4 million pixels, with professional cameras using over 6 million pixels.

When assessing digital photographs of conventional radiographs a number of points have been established. First, telephonically transmitted digital images do not differ in quality from stored images. ${ }^{15}$ Second, digital images viewed on different state-of-the-art monitors, including laptop computers, are of equal quality. ${ }^{16}$ Third, observers estimating distances from digital images displayed on monitors adjust very well to larger images, with a high degree of accuracy, despite digital images having been shown to have greater magnification distortion than conventional radiographs. ${ }^{4,17}$ Finally, digital images are likely to be viewed on monitors with background room lighting (usually fluorescent lights), and this has been shown not to affect detection of clinical information. ${ }^{18}$

One of the fundamental aspects of digital systems is the size of the pixel in the image sensor, as opposed to the silver grain emulsion size in conventional radiographs. The pixel size for the Nikon D1X camera is approximately $12 \mu \mathrm{m}$ by $12 \mu \mathrm{m}$, whereas the silver halide grain in $\mathrm{F}$ speed film is about $1.5 \mu \mathrm{m}$ by $1.5 \mu \mathrm{m}$ (personal communication with Eastman Kodak Company). Thus, the file tip of an ISO 06 file cannot be accurately recorded or viewed by a digital system with a pixel size that is bigger than the file tip, such as the Digora $(64 \mu \mathrm{m}$ by $64 \mu \mathrm{m})$. We initially used ISO 06 file sizes at working length but in most cases they proved unreadable on the conventional clinical radiographs. The tip of the ISO 08 file also proved difficult to localize at times, and was the primary reason why some of the original radiographs were deemed to be nondiagnostic by the evaluators. Nevertheless, this file size was selected to provide a rigorous test of the digitization capability of the three imaging modalities.

The low Kappa values for intraexaminer and interexaminer reliability for digital imaging modes were caused by several factors. The Kappa statistic can be strongly influenced by only a few disagreements, which can be clearly seen in the Kappa value of 0.48 for an $87 \%$ interexaminer agreement that the conventional radiographs were of diagnostic value. In a simple two-outcomes, two rater model with 40 evaluations, a Kappa value of 0.48 or lower would correspond to only five disagreements out of 40. Furthermore, evaluators with digital experience were unavailable for our study. The learning curve for new devices and techniques can be quite extensive, limiting the ability to perceive subtle differences between digital imaging modalities with similar capabilities. ${ }^{19}$ Even for the assessment of conventional radiographs it has been shown that extensive training can be necessary in order to achieve good reproducibility of observations. ${ }^{20,21}$ It is likely that greater experience with digital images and more stringent criteria would result in better Kappa values. ${ }^{22}$

The ranking of image quality by three evaluators not experienced in digitalization could bias the present data, making the digitization modes appear to be inferior. In this regard, the results need to be interpreted with some caution and it would be interesting to repeat this research with examiners experienced in digital imaging. However, our evaluators were constantly able to refer to the original radiograph when identifying features on the digital images and deciding on their diagnostic value. Thus, the diagnostic capability per se of the digital images (as opposed to the rankings) may not have been greatly influenced by bias. Furthermore, evaluators experienced in digitization may introduce a bias towards digital technology. Subconsciously, the known benefits of digital radiography in dentistry such as speed and reduced radiation exposure may outweigh any loss in resolution. Digital technology may also be sufficient for certain clinical decisions such as working length determination with a certain file size, but not for example, detection of early proximal caries. The number of radiographs used was insufficient to draw any conclusions, but future studies could be directed at making such comparisons.

The study showed similarities between the digital imaging modalities. This is because irrespective of the resolution of the camera or scanner, the resolution of the viewing screen will to a great degree determine the ultimate visual quality. The conversion of digital camera images to 256 grey scale did not seem to adversely affect the quality of the images. Ironically the slide scanner images, which had the largest file sizes and required no grey scale conversion, were often deemed to have the poorest contrast. It is unclear why this was the case, but it did result in the slide scanner being inferior in some aspects to the two digital cameras.

The original radiographs stood out by far as the best images. This is in contrast to other studies in the medical 
literature. ${ }^{8,9}$ Dental radiology deals with minute sizes and a number of closely related and superimposed mineralized structures, which can make subtle differences difficult to perceive. In addition, different methodologies may also contribute to different results. It is of interest that there was effectively no difference between a $300 \mathrm{kB}$ image and a $10 \mathrm{MB}$ image on the viewing monitor. Thus, although dental digital imaging has shown considerable progress, further improvement is still necessary. Part of this improvement will need to be directed at viewing monitors, both in terms of number of pixels and pixel size. Higher resolution screens will enable better use of modern, very

\section{References}

1. Sanderink GC, Huiskens R, van der Stelt PF, Welander US, Stheeman SE. Image quality of direct digital intraoral $x$-ray sensors in assessing root canal length. The RadioVisioGraphy, Visualix/VIXA, Sens-A-Ray, and Flash Dent systems compared with Ektaspeed films. Oral Surg Oral Med Oral Pathol 1994; 78: $125-132$.

2. Garcia AA, Navarro LF, Castello VU, Laliga RM. Evaluation of a digital radiography to estimate working length. J Endod 1997; 23: 363-365.

3. Cederberg RA, Tidwell E, Frederiksen NL, Benson BW. Endodontic working length assessment. Comparison of storage phosphor digital imaging and radiographic film. Oral Surg Oral Med Oral Pathol Oral Radiol Endod 1998; 85: 325-328.

4. Mentes A, Gencoglu N. Canal length evaluation of curved canals by direct digital or conventional radiography. Oral Surg Oral Med Oral Pathol Oral Radiol Endod 2002; 93: 88-91.

5. Naoum HJ, Chandler NP, Love RM. Conventional versus storage phosphor-plate digital images to visualize the root canal system contrasted with a radiopaque medium. J Endod 2003; 29: 349-352.

6. Friedlander LT, Love RM, Chandler NP. A comparison of phosphor-plate digital images with conventional radiographs for the perceived clarity of fine endodontic files and periapical lesions. Oral Surg Oral Med Oral Pathol Oral Radiol Endod 2002; 93: 321-327.

7. Fuge KN, Stuck AM, Love RM. A comparison of digitally scanned radiographs with conventional film for the detection of small endodontic instruments. Int Endod J 1998; 31: 123-126.

8. Larson A, Lynch DA, Zeligman B, Harlow C, Vanoni C, Thieme G, et al. Accuracy of diagnosis of subtle chest disease and subtle fractures with a teleradiology system. AJR Am J Roentgenol 1998; 170: $19-22$.

9. Schellingerhout D, Chew FS, Mullins ME, Gonzalez RG. Projected digital radiologic images for teaching: balance of image quality with data size constraints. Acad Radiol 2002; 9: 157-162.

10. Niamtu J. 3rd. Techno pearls for digital image management Dermatol Surg 2002; 28: 946-950. high resolution CCDs. This is applicable not only to digital photography, but also DDR.

In conclusion, digitizing conventional dental radiographs using current high-grade digital cameras or scanners did not produce images of diagnostic quality. Improved resolution for viewing monitors is necessary to fully harness the potential of digital technology in dentistry.

\section{Acknowledgments}

We wish to thank Professor Tom Kardos for help with using the Nikon slide scanner, and Assoc. Professor Peter Herbison for help with the statistical analysis.
11. Chong BS, Pitt Ford TR, Wilson RF. Radiological assessment of the effects of potential root-end filling materials on healing after endodontic surgery. Endod Dent Traumatol 1997; 13: 176-179.

12. Sandler J, Murray A. Manipulation of digital photographs. J Orthod 2002; 29: 189-194

13. Bengel W. Digital photography in the dental practice - an overview (II). Int J Comput Dent 2000; 3: 121-132.

14. Behle C. Photography and the operating microscope in dentistry. J Calif Dent Assoc 2001; 29: 765-771.

15. Mistak EJ, Loushine RJ, Primack PD, West LA, Runyan DA Interpretation of periapical lesions comparing conventional, direct digital, and telephonically transmitted radiographic images. J Endod 1998; 24: 262-266.

16. Cederberg RA, Frederiksen NL, Benson BW, Shulman JD. Influence of the digital image display monitor on observer performance. Dentomaxillofac Radiol 1999; 28: 203-207.

17. Versteeg KH, Sanderink GC, van Ginkel FC, van der Stelt PF. Estimating distances on direct digital images and conventional radiographs. J Am Dent Assoc 1997; 128: 439-443.

18. Cederberg RA, Frederiksen NL, Benson BW, Shulman JD. Effect of different background lighting conditions on diagnostic performance of digital and film images. Dentomaxillofac Radiol 1998; 27: 293-297.

19. Parry BR, Williams SM. Competency and the colonoscopist: a learning curve. Aust N Z J Surg 1991; 61: 419-422.

20. Goldman M, Pearson AH, Darzenta N. Endodontic success-who's reading the radiograph? Oral Surg Oral Med Oral Pathol 1972; 33: $432-437$.

21. Saunders MB, Gulabivala K, Holt R, Kahan RS. Reliability of radiographic observations recorded on a proforma measured using inter- and intra-observer variation: a preliminary study. Int Endod J 2000; 33: $272-278$

22. Eckerbom M, Andersson JE, Magnusson T. Interobserver variation in radiographic examination of endodontic variables. Endod Dent Traumatol 1986; 2: 243-246. 